\title{
Who ordered the anti-de Sitter tangent group?
}

\author{
Ali H. Chamseddine ${ }^{a, b}$ and Viatcheslav Mukhanov ${ }^{c, d, e}$ \\ ${ }^{a}$ Physics Department, American University of Beirut, \\ Beirut, Lebanon \\ ${ }^{b}$ Institut des Hautes Études Scientifiques - Le Bois-Marie 35, \\ route de Chartres, 91440 Bures-sur-Yvette, France \\ ${ }^{c}$ Theoretical Physics, Ludwig Maxmillians University, \\ Theresienstr. 3\%, 80333 Munich, Germany \\ ${ }^{d}$ LPT de l'Ecole Normale Superieure, Chaire Blaise Pascal, \\ 24 rue Lhomond, 75231 Paris cedex, France \\ e MPI for Physics, \\ Foehringer Ring, 6, 80850, Munich, Germany \\ E-mail: chams@aub.edu.lb, viatcheslav.mukhanov@physik.uni-muenchen.de
}

AbSTRaCt: General relativity can be unambiguously formulated with Lorentz, de Sitter and anti-de Sitter tangent groups, which determine the fermionic representations. We show that besides of the Lorentz group only anti-de Sitter tangent group is consistent with all physical requirements.

KEYwORDS: Supersymmetry and Duality, Classical Theories of Gravity

ARXIV EPRINT: 1308.3199 
The existence of fermions forces us to consider the tangent group $\mathrm{SO}(3,1)$, or equivalently the SL $(2, \mathbb{C})$ group, in General Relativity. Promoting the global Lorentz invariance of the Dirac action to a local one is achieved by introducing the spin-connection as the gauge field of the tangent group. The vierbein, which is the square root of the metric, is the soldering form connecting coordinate basis vectors to orthogonal tangent vectors. Metricity condition is expressed as the vanishing of the covariant derivative on the vierbein with respect to both the spin-connection and the symmetric affine connection. For the Lorentz tangent group the resulting system of equations is enough to determine both the spin-connection and the symmetric affine connection in terms of the vierbein and its first derivatives unambiguously. It was established long ago that in this case the scalar curvature constructed of the metric manifold is equivalent to the scalar curvature constructed in terms of the spin-connection. Hence, in the presence of spinors one can formulate gravitational interactions in terms of the vierbein and (dependent) spin-connections and we are assured that the corresponding curvature scalar is equivalent to the metric dependent scalar curvature [1-3]. It came as a surprise that the metricity conditions can also be unambiguously solved for the de Sitter and anti-de Sitter tangent groups and the curvature invariants with respect to the tangent group gauge fields are identical to the metric curvature in these cases [4]. To find the coupling to the spinors for these tangent groups one must first determine the irreducible spinor representations of the groups $\mathrm{SO}(4,1)$ or $\mathrm{SO}(3,2)$ and check when it is possible to construct a realistic model for the quarks and leptons.

Let us define the inverse soldering forms $e_{A}^{\mu}$ where $\mu=0,1,2,3$ is a space-time index and $A=a, 4$ with $a=0,1,2,3$ is a tangent space index. The inverse metric $g^{\mu \nu}$ is then given by $g^{\mu \nu}=e_{A}^{\mu} \eta^{A B} e_{B}^{\nu}$ with $\eta^{A B}=\left(\eta^{a b}, \eta^{44}=\epsilon\right)$, where $\eta^{a b}=\operatorname{diag}(1,-1,-1,-1)$ is the Minkowski metric, and $\epsilon=-1$ for the de Sitter tangent group $\operatorname{SO}(4,1)$ and $\epsilon=+1$ for anti-de Sitter group $\mathrm{SO}(3,2)$. The metricity condition is then

$$
\nabla_{\mu} e_{A}^{\nu} \equiv \partial_{\mu} e_{A}^{\nu}+\omega_{\mu A} B e_{B}^{\nu}+\Gamma_{\mu \rho}^{\nu} e_{A}^{\rho}=0,
$$

where $\omega_{\mu A}{ }^{B}$ is the gauge connection of the groups $\mathrm{SO}(4,1)$ or $\mathrm{SO}(3,2)$ and $\Gamma_{\mu \rho}^{\nu}$ is the symmetric affine connection of the diffeomorphism group satisfying $\Gamma_{\mu \rho}^{\nu}=\Gamma_{\rho \mu}^{\nu}$. The eighty conditions (1) can be solved to determine the eighty fields $\omega_{\mu A}{ }^{B}$ and $\Gamma_{\mu \rho}^{\nu}$ in terms of $e_{A}^{\nu}$ and the first derivatives $\partial_{\mu} e_{A}^{\nu}$. In particular the affine connection is given by the familiar Christoffel symbols

$$
\Gamma_{\mu \rho}^{\nu}=\frac{1}{2} g^{\nu \sigma}\left(\partial_{\mu} g_{\sigma \rho}+\partial_{\rho} g_{\mu \sigma}-\partial_{\sigma} g_{\mu \rho}\right)
$$

where $g_{\mu \nu}$ is the inverse of $g^{\mu \nu}$. The curvature of the spin-connection $\omega_{\mu A}{ }^{B}$ is given by

$$
R_{\mu \nu}^{A B}(\omega)=\partial_{\mu} \omega_{\nu}^{A B}-\partial_{\nu} \omega_{\mu}^{A B}+\omega_{\mu}^{A C} \omega_{\nu C}{ }^{B}-\omega_{\nu}^{A C} \omega_{\mu C}{ }^{B},
$$

and is related to the curvature of the affine connection by the relations

$$
\begin{aligned}
R(\omega) & =e_{A}^{\mu} R_{\mu \nu}^{A B}(\omega) e_{B}^{\nu}=-R_{\sigma \mu \nu}^{\nu}(\Gamma) e^{\sigma A} e_{A}^{\mu} \\
& =R_{\sigma \nu \mu}^{\nu}(\Gamma) g^{\sigma \mu}=R(\Gamma),
\end{aligned}
$$

where

$$
R_{\sigma \mu \nu}^{\rho}(\Gamma)=\partial_{\mu} \Gamma_{\nu \sigma}^{\rho}-\partial_{\nu} \Gamma_{\mu \sigma}^{\rho}+\Gamma_{\mu \kappa}^{\rho} \Gamma_{\nu \sigma}^{\kappa}-\Gamma_{\nu \kappa}^{\rho} \Gamma_{\mu \sigma}^{\kappa} .
$$


Thus, although the scalar curvature of the spin-connection is a function of $e_{A}^{\mu}$, it is expressible as function of the metric $g^{\mu \nu}$ only because of the invariance under the local $\mathrm{SO}(4,1)$ or $\mathrm{SO}(3,2)$ gauge transformations. Thus, in the absence of matter couplings the gravity formulations in terms of the metric tensor, the vierbein $e_{a}^{\mu}$ for the Lorentz tangent group $\mathrm{SO}(3,1)$, the vielbein $e_{A}^{\mu}$ for the tangent groups $\mathrm{SO}(4,1)$ or $\mathrm{SO}(3,2)$ are all equivalent. To find the physical consequences of the choice of tangent group let us consider matter couplings.

It is well known that the bosonic fields do not "feel" the tangent group. The relevant fundamental couplings in this case are those of scalars and vectors. In the case of the Lorentz tangent group $\mathrm{SO}(3,1)$ there is one to one correspondence between vectors with respect to the diffeomorphism transformations and to the local tangent group gauge transformations. For the other tangent groups $\mathrm{SO}(4,1)$ and $\mathrm{SO}(3,2)$ a five dimensional vector $V_{A}$ with respect to the tangent group projects into a diffeomorphism vector $A_{\mu}$ and a scalar $\phi$

$$
V_{A}=e_{A}^{\mu} A_{\mu}+n_{A} \phi
$$

where $n_{A}$ is a unit vector orthogonal to $e_{A}^{\mu}$

$$
n^{A} e_{A}^{\mu}=0, \quad n_{A} n^{A}=\varepsilon,
$$

with $\varepsilon=1$ for $\mathrm{SO}(3,2)$ and $\varepsilon=-1$ for $\mathrm{SO}(4,1)$ [4]. Therefore, there is no obvious advantages in formulating the vector interactions for the tangent group vectors because this is a reducible representation and the resulting action does not have any extra symmetries. Moreover, only the tangent group $\mathrm{SO}(4,1)$ gives the correct sign for the kinetic energy of the extra scalar field which inevitably emerges in this case. Hence it seems that the only relevant symmetry for the bosonic fields is the diffeomorphism symmetry.

The situation is different for the fermions as those ones are defined as representations of the tangent group. For the $\mathrm{SO}(3,1)$ tangent group the spinors transform under local gauge transformations as

$$
\delta \psi_{\alpha}=\frac{1}{4} \Lambda_{a b}\left(\gamma^{a b}\right)_{\alpha}^{\beta} \psi_{\beta}, \quad a=0,1,2,3,
$$

where $\Lambda_{a b}=-\Lambda_{b a}$ are the gauge parameters and $\gamma^{a b}=\frac{1}{2}\left(\gamma^{a} \gamma^{b}-\gamma^{b} \gamma^{a}\right)$. We adopted here the notation where the Dirac matrices satisfy $\left\{\gamma^{a}, \gamma^{b}\right\}=2 \eta^{a b}$ with

$$
\eta^{a b}=\operatorname{diag}(1,-1,-1,-1) .
$$

The transformations preserve either Weyl or Majorana condition on the spinors but not both ones. If the Weyl condition $\gamma_{5} \psi=\psi$ is imposed, where $\gamma_{5}=i \gamma^{0} \gamma^{1} \gamma^{2} \gamma^{3}$ satisfies $\left(\gamma_{5}\right)^{2}=1$, then

$$
\delta\left(\gamma_{5} \psi\right)_{\alpha}=\frac{1}{4} \Lambda_{a b}\left(\gamma^{a b}\right)_{\alpha}^{\beta}\left(\gamma_{5} \psi\right)_{\beta} .
$$

Alternatively, imposing the Majorana condition $\psi_{\alpha}=C_{\alpha \beta}\left(\bar{\psi}^{\beta}\right)^{T}$ where $C$ is the charge conjugation matrix, is preserved by the gauge transformations because it is possible to find Dirac matrices, satisfying the symmetry condition $\left(\gamma^{a b} C\right)_{\alpha \beta}=\left(\gamma^{a b} C\right)_{\beta \alpha}[8]$. We note that the Standard Model is formulated in terms of chiral leptons and quarks, while the 
minimally supersymmetric standard model is formulated in terms of Majorana fermions. This is possible because all physical fermions correspond to Dirac spinors which acquire their mass by coupling left-handed spinors to the right handed ones, which in turn can also be decomposed as the complex sum of two Majorana spinors. Although direct Majorana mass terms are possible, these would break the $\mathrm{SU}(3) \times \mathrm{SU}(2) \times \mathrm{U}(1)$ gauge symmetries, except for the right-handed neutrino, which is desirable.

Repeating this analysis for the $\mathrm{SO}(4,1)$ tangent group, the gauge transformations of the spinors are now

$$
\delta \psi_{\alpha}=\frac{1}{4} \Lambda_{A B}\left(\Gamma^{A B}\right)_{\alpha}^{\beta} \psi_{\beta}, \quad A=a, 4,
$$

where the Dirac matrices $\Gamma^{A}$ satisfy $\left\{\Gamma^{A}, \Gamma^{B}\right\}=2 \eta^{A B}$ where

$$
\eta^{A B}=\operatorname{diag}(1,-1,-1,-1,-1) \text {. }
$$

A convenient representation is to take $\Gamma^{a}=\gamma^{a}$ and $\Gamma^{4}=i \gamma_{5}$. In this case it is obvious that the Weyl condition $\gamma_{5} \psi=\psi$ is not preserved by the gauge transformation. In addition, the Majorana condition $\psi_{\alpha}=C_{\alpha \beta}\left(\bar{\psi}^{\beta}\right)^{T}$ is also not preserved by the gauge transformations because

$$
\left(i \gamma_{5} \gamma_{a} \psi\right)=-C{\overline{\left(i \gamma_{5} \gamma_{a} \psi\right)}}^{T} .
$$

The minus sign is a consequence of the signature $\eta^{44}=-1$ which forces $\Gamma^{4}$ to be equal to $i \gamma_{5}$. This also implies that the Majorana condition could be imposed for the tangent group $\mathrm{SO}(3,2)$. Thus, when the tangent group is $\mathrm{SO}(4,1)$ only complex Dirac spinors are allowed. To construct realistic models, each of the fermionic representations must be complex, and for the Standard Model the lepton doublet would have both right and left-handed components, and similarly for the leptonic singlets. This creates the problem of mirror fermions, as the physical particle formed by coupling the Higgs field to the leptonic doublets and singlet, will have a partner with the same mass but formed from the opposite chiralities. For example if we denote the Dirac leptonic doublet by $l=\left(\begin{array}{c}\nu_{e} \\ e\end{array}\right)$, the Dirac singlet by $\widetilde{e}$ and the Higgs field by $H$ the fermionic terms are then given by

$$
\begin{aligned}
& i \bar{l} e_{A}^{\mu} \Gamma^{A}\left(\partial_{\mu}+\frac{1}{4} \omega_{\mu}^{B C} \Gamma_{B C}\right) l+i \overline{\widetilde{e}} e_{A}^{\mu} \Gamma^{A}\left(\partial_{\mu}+\frac{1}{4} \omega_{\mu}^{B C} \Gamma_{B C}\right) \widetilde{e} \\
& +f\left(\bar{l} H \widetilde{e}+\overline{\widetilde{e}} H^{*} l\right)+m(\bar{l} l+\overline{\widetilde{e}} \widetilde{e}) .
\end{aligned}
$$

When $H$ gets an expectation value, a mass term results of the form

$$
f \mu\left(\bar{e}_{L} \widetilde{e}_{R}+\bar{e}_{R} \widetilde{e}_{L}+\overline{\widetilde{e}}_{L} e_{R}+\overline{\widetilde{e}}_{R} e_{L}\right)+m\left(\bar{e}_{L} e_{R}+\bar{e}_{R} e_{L}+\overline{\widetilde{e}}_{L} \widetilde{e}_{R}+\overline{\widetilde{e}}_{R} \widetilde{e}_{L}\right)
$$

which shows that we have two massive electrons formed from combinations of $e_{L}, \widetilde{e}_{R}$ and $e_{R}, \widetilde{e}_{L}$. One must then tune one combination to have a small mass and identified with the electron while the other combination would be heavy. In the Standard Model because of quadratic divergencies in the Higgs sector, the fermionic masses must be tuned to be low, which is the hierarchy problem. In this case fine tuning is needed between the two fermion 
masses to keep one combination low, in addition to the hierarchy problem resulting from quadratic divergencies. In this respect, the tangent group $\mathrm{SO}(4,1)$ seems to be less natural than the $\mathrm{SO}(3,1)$ tangent group.

We can easily see that when the tangent group is $\mathrm{SO}(3,2)$ we can impose the Majorana condition. It is known that the algebra $\mathrm{SO}(3,2)$ is isomorphic to the algebra $S P(2,2)$ with generators $M_{\alpha \beta}=M_{\beta \alpha}$ satisfying the commutation relations [5-7],

$$
\left[M_{\alpha \beta}, M_{\gamma \delta}\right]=C_{\alpha \gamma} M_{\beta \delta}+C_{\beta \gamma} M_{\alpha \delta}+C_{\alpha \delta} M_{\beta \gamma}+C_{\beta \delta} M_{\alpha \gamma},
$$

where $C_{\alpha \beta}=-C_{\beta \alpha}$ is the charge conjugation matrix. Connection between the generators of the two groups is made through the identification $M_{\alpha \beta}=\frac{1}{4} M_{A B}\left(\Gamma^{A B} C\right)_{\alpha \beta}$, where $A=a, 4$ and $\Gamma^{a}=i \gamma_{5} \gamma^{a}, \Gamma^{4}=\gamma_{5}$. The gauge transformations then take the simple form $\delta \psi_{\alpha}=M_{\alpha}^{\beta} \psi_{\beta}$ where $M_{\alpha}^{\beta}=M_{\alpha \gamma} C^{\gamma \beta}$ and thus preserve the Majorana condition. The vielbein $e_{A}^{\mu}$ are in the antisymmetric representation of $\operatorname{SP}(2,2)$

$$
\left(e^{\mu}\right)_{\alpha \beta}=-\left(e^{\mu}\right)_{\beta \alpha}=\left(\Gamma^{A} C\right)_{\alpha \beta} e_{A}^{\mu}
$$

The fermionic action can thus be written in terms of the matrices $\left(e^{\mu}\right)_{\alpha \beta}$ and $\left(\omega_{\mu}\right)_{\alpha \beta}=$ $\frac{1}{4} \omega_{\mu}^{A B}\left(\Gamma^{A B} C\right)_{\alpha \beta}$

$$
i \bar{\psi} e^{\mu}\left(\partial_{\mu}+\omega_{\mu}\right) \psi
$$

and the commutator of the covariant derivatives $D_{\mu}=\partial_{\mu}+\omega_{\mu}$ gives

$$
\begin{aligned}
{\left[D_{\mu}, D_{\nu}\right]_{\alpha \beta} } & =R_{\mu \nu \alpha \beta} \\
& =\frac{1}{4} R_{\mu \nu}{ }^{A B}\left(\Gamma_{A B} C\right)_{\alpha \beta}
\end{aligned}
$$

We conclude that there is no obstruction to construct the Standard Model using the tangent group $\mathrm{SO}(3,2)$ and using Majorana spinors in the same way the minimally supersymmetric standard model is built. The bosons transform under the diffeomorphism group and do not feel the tangent group. In this respect there is no advantage in curved spaces of using the Lorentz group instead of the anti-de Sitter group. This leads to the puzzling question of whether there is any significance to the ambiguity in having two possibilities for the tangent group.

\section{Acknowledgments}

The work of AHC is supported in part by the National Science Foundation Phys-0854779, and Phys-1202671. The work of VM is supported by "Chaire Internationale de Recherche Blaise Pascal financée par l'Etat et la Région d'Ile-de-France, gérée par la Fondation de l'Ecole Normale Supérieure", by TRR 33 "The Dark Universe" and the Cluster of Excellence EXC 153 "Origin and Structure of the Universe".

Open Access. This article is distributed under the terms of the Creative Commons Attribution License which permits any use, distribution and reproduction in any medium, provided the original author(s) and source are credited. 


\section{References}

[1] R. Utiyama, Invariant theoretical interpretation of interaction, Phys. Rev. 101 (1956) 1597 [INSPIRE].

[2] T. Kibble, Lorentz invariance and the gravitational field, J. Math. Phys. 2 (1961) 212 [INSPIRE].

[3] S. Weinberg, Generalized theories of gravity and supergravity in higher dimensions, in Proceeding of fifth workshop on grand unification, K. Kang, H. Fried and P. Frampton eds., World Scientific, Singapore (1984) [INSPIRE].

[4] A.H. Chamseddine and V. Mukhanov, Gravity with de Sitter and unitary tangent groups, JHEP 03 (2010) 033 [arXiv: 1002.0541] [INSPIRE].

[5] A.H. Chamseddine and P.C. West, Supergravity as a gauge theory of supersymmetry, Nucl. Phys. B 129 (1977) 39 [InSPIRE].

[6] S. MacDowell and F. Mansouri, Unified geometric theory of gravity and supergravity, Phys. Rev. Lett. 38 (1977) 739 [Erratum ibid. 38 (1977) 1376] [INSPIRE].

[7] A.H. Chamseddine, Massive supergravity from spontaneously breaking orthosymplectic gauge symmetry, Annals Phys. 113 (1978) 219 [INSPIRE].

[8] J. Scherk, Extended supersymmetry and extended supergravity theories, in Recent developments in gravitation, Cargèse 1978, M. Lévy and S. Deser eds., Plenum Press, U.S.A. (1978) [INSPIRE]. 